\title{
Differences in the Transference of Humor and Personification in Advertisement Translation
}

\author{
Ying Cui \\ City University of Hong Kong \\ Qingmei Wang \\ Ludong Unversity
}

\begin{abstract}
This study analyses the transference of humor in translating advertising texts and, based on research in a prior study, proposes that the transference of humor is quite flexible in the practice of advertisement translation. The humorous expressions in original texts may be faithfully translated, or deleted, or in some cases humor may be added in translated texts. Compared with such flexible ways of dealing with humor, the translation of personifycation in our corpus is relatively more faithful. In this paper, we are trying to figure out the implications behind the difference in terms of the treatment of humor and personification in advertisement translation. Similar to the former study, here we will also take the presupposition perspective. When the personification is reproduced faithfully in translations, it can be inferred that translators' presuppositions about the target audience's potential perception are the same with those held by the original writers about their targeted readers. This point in turn implies that the original and target cultures are correspondent in those aspects. Similarly, the unfaithful or flexible treatment of humor implies the opposite. In our research
\end{abstract}


48 Differences in the Transference of Humor and Personification

we mainly focus on translation between Chinese and English.

Keywords: humour, personification, translation, advertising, discourse

\section{Introduction}

In this paper, we intend to figure out the possible cultural implications behind the different ways of dealing with humor and personification in translating advertising texts. The corpus we are working on is composed of 98 bilingual advertising texts collected from four sources, including the internet, "An Anthology of Selected English Advertisements" (Wu \& Hu 1998), "Advertising English: 3000 examples" (Cui 1993), and bilingual advertisements mentioned in papers on advertisement translation published between 2006 and 2007 as retrieved in Wanfang Database and China Academic Journals Full-text Database (Beijing Site). Observation of the examples within our data shows that humor and personification are dealt with in very different ways. While humor is transferred with much flexibility, personification is very often reproduced faithfully.

This research mainly involves Chinese and English, we have focused on Wanfang Database (1998-2008) and China Academic Journals Full-text Database (1998-2008, Beijing Site) in order to review the study on humor, personification and advertisement translation. However, we are not able to find any relevant papers or other monographs on this subject matter. There are separate studies on humor or personification in advertising texts or their translation, but there is no research concentrating on the comparison between the ways of dealing with humor and personification in translation. This is one of the reasons why we have taken on this study, exploring the translation of humor and personification from the 
presupposition angle.

This paper is composed of five parts. In the first section, we will clarify the definitions of humour and personification. In the second section, we will briefly restate what we have done on the translation of humour in advertising texts. In the third section, we are going to elaborate on the handling of personification in translating advertisements. The fourth section will illustrate the concept of presupposition as explored in linguistics and applied in this study. The fifth section will provide a comparison between the dealing of humour and personification, and an exploration into the implications from the presupposition perspective.

\section{Notions of Humor and Personification}

Personification is defined as "a figure of speech in which inanimate objects or abstractions are endowed with human qualities or are represented as possessing human form" (Kingsoft Powerword 2007). As to humour, it is defined in terms of various layers of meaning, and the implications of humour that are relevant to our research cover the following: the quality that makes something laughable or amusing; funniness; that which is intended to induce laughter or amusement. We can infer that, in some cases, personification may also be able to produce the effect of humour, as we will show later. However, in this study, we are not focusing on such overlap between the two; instead, we treat them as separate entities and we are trying to find the difference of dealing with them in translation.

\subsection{Research on Humor}

In our paper entitled "Humour in Advertisement Translation-With special reference to translation between Chinese and English," 
50 Differences in the Transference of Humor and Personification

published in English in the World----Special issue: Humor and aspects related to linguistics and translation, cultural studies and literature 2010, 419-453, we studied the same corpus mentioned in the Introduction. From that work, in this section we will list three major ways of handling humor in translation as summarized in the paper.

\subsection{Examples of Translating Humor}

In our data, 34 examples have a humorous flavor. For 25 of these examples, the source texts are translated in a flexible way. For 10 of them, the humour is realized via personification, and 9 of these advertisements are faithfully translated. In terms of handling humorous expressions, there are mainly three strategies in translating advertising texts, and for each of them we will provide an example.

Firstly, while original texts do not contain humorous expressions, humour may be added in their translations. See the following example:

(1) We know exactly how to sell eggs. 不求完美, “蛋” 求鲜美

(bu qiu wan mei, dan qiu xian mei; we do not seek for perfection, but seek for the freshness of eggs.)

In example (1), there is not any humorous effect in the English version. Instead, it is a guarantee of good service and a promise to provide consumers with good-quality eggs. In the Chinese version, however, there is a humorous element, which lies in the Chinese character for eggs, “蛋 (dan, egg)”. It is homonym to “但(dan, but)”, which means both "but" and "only" in Chinese. Therefore, the Chinese version has several implied meanings. First, the seller promises to provide fresh eggs; second, even if the eggs do not look perfect, they are of good quality and they are fresh; third, far from 
being pretentious to say that the eggs are perfect, the seller is practical in being devoted to serving consumers with good eggs. What is more, as just noted, the Chinese version provides some humorous pleasure for readers via the word play of “蛋 (dan, egg)" and “但(dan, but)".

Secondly, for original texts that do have humorous expressions, such humour may be deleted in their translations due to various cultural or linguistic factors. See the following example:

(2) Deliciously simple. Simply delicious.

美味便捷，美味至极

(mei wei bian jie, mei wei zhi ji; delicious and convenient, delicious to the full.)

In example (2), which is an advertisement for fast food, the English version is composed of a word play on two words, namely delicious and simple. The two sentences both use these two words, just in different order, and in different adjective or adverb forms. Besides the humour as realized via such surface form, the two lines also convey different layers of implication. "Deliciously simple" is saying that although the food is prepared in a simple manner, the taste is delicious. "Simply delicious" implies that the food is definitely and absolutely delicious. In this way, the expression can produce some humorous effects via the word play while transferring the nuanced meaning in the two lines. In the Chinese text, although the meaning of the two English lines is reproduced, the word play cannot be directly delivered. Some attention has been paid to the surface structure, and the expression of “美味(mei wei, good taste)” is repeated in the two lines, which are quite rhythmic and balanced in the sense that each line has four Chinese characters; however, we cannot detect any humorous effect. Such loss of humour is mainly due to characteristics of the Chinese language, because Chinese characters always appear in the same form, whether they are 
adjectives, adverbs, verbs or nouns.

Thirdly, there are occasions where humour in original texts can be successfully transferred to translated texts, with the help of various linguistic or cultural coincidences. We list a few examples as selected from our data:

(3) It says what it does. It does what it says.

言行一致, 说到做到

(yan xing yi zhi, shuo dao zuo dao; We practise what we preach, and we are the equal of our word.)

In example (3), the English version is composed of two paralleled and balanced lines, which employ the same words just in different orders. Aside from such humorous effects in terms of the form, we can also find humorous effect that lies with the meaning. It is an advertisement about gargle water, but the tone suggests that the words are describing a person who is honest, reliable, responsible and upright. This kind of personification is quite vivid and effective. The Chinese version cannot reproduce the word play that is applied in the English text, but the two lines are paralleled too, with four Chinese characters in each line. Besides, the meaning is conveyed faithfully. “言行一致, 说到做到(yan xing yi zhi, shuo dao zuo $d a o$; We practise what we preach, and we are the equal of our word)" is also describing a responsible person.

\subsection{Summary of Translating Humour}

Illustration of the three different strategies in handling humor shows that the translation of humorous expressions in advertising texts is quite flexible. As in many cases, humor is realized and expressed via word play or the linguistic surface, the linguistic differences between Chinese and English determine that the humor in the original language cannot be reproduced in the target language. 
Similarly, humorous effects that cannot be created in the original language may turn out to be possible in the target language. Therefore, what translators usually do is make flexible and creative use of the language that is translated into. The target linguistic characteristics may be made use of to compose humorous expressions which are probably different from those in the original texts. In one word, since humor depends to a great degree on the linguistic features of the languages that is involved in translation, different ways of handling humor are used, as long as effective readings can be transferred. Compared with the various ways we have detected in translating humorous expressions, the handling of personification is quite faithful. In most cases, personification is reproduced faithfully in translated texts, which we will illustrate with specific examples in the following section.

\section{Translation of Personification}

As noted earlier, within our data, there are altogether 10 advertisements using personification, and out of these 10 examples, translations of 9 advertisements have reproduced the original personification faithfully. Compared with what we have said about humor, the translation of personification is much more direct and faithful. See the following examples of personification.

(4) He laughs best, who runs longest.

谁跑得最长, 谁笑得最好

(shui pao de zui chang, shui xiao de zui hao; who runs longest, who laughs best. )

(5) Unlike me, my Rolex never needs a rest.

与我不同, 我的劳力士手表从来不需要休息

(yu wo bu tong, wo de lao li shi shou biao cong lai bu 
54 Differences in the Transference of Humor and Personification

xи уао хіи xi; different from me, my Rolex watch never needs a rest.)

(6) To the ends of the earth/and to the top of the world./Only two of us have made it./... It's the only thing that's been on all the trips with me and it never once let me down.

走遍天涯海角， 登上世界之巅，你我相伴，踏遍万 水千山

(zou bian tian ya hai jiao, deng shang shi jie zhi dian, ni wo xiang ban, ta bian wan shui qian shan; walking to the ends of the earth, climbing to the top of the world, we are always together, to share the trials of this long journey.)

Example (4) is an advertisement about tyres. In both the English and Chinese versions, tyres are compared to human beings. An English idiom is adapted here. "He laughs best who laughs last" is changed into "He laughs best who runs longest". Originally the idiom is to remind people of the un-stability of situation, and a temporarily good thing may turn out to be bad in the end. The English version is translated into Chinese in a quite faithful way. Here in both versions of the slogan, the long life and durability of tyres is emphasized. In example (5), the Rolex watch is compared to a friend, who is strong and dependable and does not even need a rest. The Chinese version is equivalent to the English one, and it also keeps this personification. Besides, the wording of the Chinese text is very much correspondent to that of the original. Example (6) is also an advertisement about Rolex watch, which is personified as a loving friend, who can accompany consumers to go through all the trials of life and leave them romantic memories. The Chinese version keeps this personification, although it uses different wording in order to express the ideas in balanced and paralleled phrases making use of the characteristics of the Chinese language. 


\section{Presupposition}

Having listed the different ways of dealing with humor and personification in translating advertising texts in the above two sections, we will explore the reasons or implications behind this phenomenon. Before coming to that, we need to clarify the definition of presupposition, which we are going to take as the main perspective to explore the phenomenon just mentioned. In the following section we will provide a brief review on the understanding of presupposition in the linguistics field and the definition we are applying in this study.

\subsection{Overview of Presupposition}

Generally speaking, there are two approaches to presupposition in linguistics, namely semantic and pragmatic approaches. The semantic approach is centered on the concept of truth, which, in standard mathematical logic, is conceived as a relation between a sentence and the world (Keenan 1998: 8). Semantic presupposition refers to a semantic relation between sentences or propositions, which is independent of beliefs of speaker and hearer, background knowledge or other contextual factors (Sandt 1988: 13). We can describe semantic presupposition as "A presupposes B if the truth of $\mathrm{B}$ is a condition for the semantic value of A to be true or false" (Beaver 2001: 8-9). In contrast, the pragmatic approach towards presupposition has mainly drawn upon the theory of speech acts and that of conversational implicatures (Marmaridou 2000: 136). Specific viewpoints concerning pragmatic presupposition are various, each with its focus, but the pragmatic conception of presupposition is always closely connected with the notion that presupposition is a propositional attitude instead of a semantic relation. In Caffi's words, "pragmatic presuppositions not only concern knowledge, whether true or false: they concern expectations, 
56 Differences in the Transference of Humor and Personification

desires, interests, claims, attitudes towards the world, fears etc"(Caffi, as cited in Mey 2001: 186). It is safe to claim that pragmatic presupposition has been located in a wider communicative setting covering such notions as speaker, hearer, context, belief, appropriateness and mutual knowledge (Sergerdahl 1996: 190).

Despite all the arguments between the two approaches, we believe that presupposition is of both semantic and pragmatic nature. As also mentioned by other scholars, on the one hand, the use of certain linguistic expressions implies the true belief of the utterer concerning the existence of the relevant referents, and this aspect is more of a semantic one; on the other hand, sentences are instruments used intentionally by agents in specific contexts, and the agents and addressees are presumed to have particular beliefs and attitudes, hence the pragmatic aspect to presupposition (Green 1989: 112-113). So the two approaches are not in conflict. Instead, they are "explications of related but different ideas", and "any semantic presupposition of a proposition expressed in a given context will be a pragmatic presupposition of the people in that context" (Stalnaker, as cited in Sergerdahl 1996: 189). In other words, "a semantic presupposition of a sentence is a pragmatic presupposition of the users of the sentence" (Sandt 1998: 26). We are seeking for the application of presupposition covering both aspects, not only presuppositions as triggered by certain expressions (semantic presupposition) but also the contextual influence (pragmatic presupposition).

\subsection{Presuppositions in this Study}

In the present study, which explores the translation of advertising discourse, the target context is especially important, for advertising texts have a specific purpose of persuading the target audience, who are involved in and influenced by their context. When translating advertising texts, normally translators will consider the target 
audience's possible reaction within their specific context, and in this regard we will consider context as the source of presuppositions.

Similar illustration regarding the relationship between presupposition and context can be found in Givón (1989: 135-137), where presupposed information is traced back to the major subdivisions of context. The major subdivisions of context are openended; however, three foci under which specific categories get grouped are "highly stable and well attested in the traditional linguistic literature" (Givón 1989: 74). First, the generic focus covers the shared world and culture, and refers to something universal to a great degree and remains the same across different cultures. It comprises on the one hand knowledge and beliefs concerning the real world, and on the other hand people's ways and capacities to make sense of the world. For illustration, people from different cultures have the same knowledge about day and night, except for those who live in the polar regions who may know only "day season" (or polar day in the technical term) and "night season"(or polar night in the technical term). Second, the deictic focus refers to the immediate communicative situation. It covers what can be known about the speech situation, social-personal relations between participants, including their respective conditions such as status, power, obligations, needs and expectations, and goals of communication. So in a specific advertising text, what needs are mainly addressed is determined by the situational context. Third, the discourse focus refers to the shared prior text. It covers not only specific words or phrases, but also the flexible and creative usage of language in both the original text and its translation. In the following section, we will explore the presuppositions behind the three ways of dealing with humour and personification with reference to this contextual framework. 


\section{Analysis of Humor}

Detailed analysis of example (1), (2) and (3) can be found in our paper "Humour in Advertisement Translation----With special reference to translation between Chinese and English". Here we will briefly restate the presuppositions we have identified in these examples. ST refers to source text, and TT target text. The sign " $>>$ " means "presuppose".

Example (1)

ST:

(a) Generic context: There is some general requirement for the quality of eggs on the part of consumers. Sellers should offer adequate goods. Consumers are in the position to be served. $>>$ Sellers are presupposed to offer fresh eggs to consumers who are in the position to be served.

(b) Situational context: The seller knows how to provide good service to consumers and implies to them that their needs for fresh eggs will be met. >> Consumers have needs for fresh, good-quality eggs.

(c) Discourse context: The word "exactly" is to emphasize that maybe many sellers to know how to sell eggs, but this seller knows better and can provide better eggs. >> Audience understands the implication of the word "exactly" and the promise in the slogan.

TT:

(d) Generic context: There is some general requirement for the quality of eggs on the part of consumers. Sellers are supposed to offer adequate goods. Consumers are in the position to be served. $>>$ Sellers are supposed to offer fresh eggs to consumers who are in the position to be 
served.

(e) Situational context: The seller is not promising perfection of eggs, but freshness. > Consumers have needs for fresh, good-quality eggs, and they appreciate honesty of sellers.

(f) Discourse context: The pronunciations of "egg" and "but" or "only" in Chinese are the same. >> Audience understands the implication of the pun, and the promise and humor that is conveyed.

Example (2)

ST:

(a) Generic context: The preparation of fast food is simple, and the taste is good. $>$ People are expected to appreciate such merits as good taste and simple preparation.

(b) Situational context: As it is advertising fast food, the merits of fast food are given prominence. $>>$ People are busy in daily life, so they are pressed in terms of time. Their needs for saving time are realized by this fast food. What is more, they also need tasty food, and the fast food advertised can also satisfy their needs.

(c) Discourse context: The same words are employed in the two lines, just in different orders and forms. $>$ Such design is supposed to be able to produce humorous effects and appreciated by audience.

TT:

(d) Generic context: The preparation of fast food is simple, and the taste is good. >> People are expected to appreciate such merits as good taste and simple preparation.

(e) Situational context: As it is advertising fast food, the 
merits of fast food are given prominence. $>>$ People are busy in daily life, so they are pressed in terms of time. Their needs for saving time are realized by this fast food. What is more, they also need tasty food, and the fast food advertised can also satisfy their needs.

(f) Discourse context: The two lines are put in balanced and paralleled form. The implication of good taste is repeated. > $>$ The balanced and paralleled wording is expected to be more easily remembered and appreciated by the Chinese audience.

Example (3)

ST:

(a) Generic context: Keeping one's promise is appreciated, and to practice what is preached is important. $>>$ Although it is only an advertisement, consumers still expect honesty and responsibility.

(b) Situational context: Personifying the product as a person and giving such promise. $\gg$ Audience may have such doubt as whether the advertisement praising the product is true or not.

(c) Discourse context: Same words are used in each line, just in different orders. The two lines are also paralleled. >> Readers are supposed to appreciate the aesthetic value in such desire and the humor in the personification.

TT:

(d) Generic context: Keeping one's promise is appreciated, and to practice what is preached is important. $>>$ Although it is only an advertisement, consumers still expect honesty and responsibility.

(e) Situational context: Personifying the product as a person and giving such promise. $>>$ Audience may have such 
doubt as whether the advertisement praising the product is true or not.

(f) Discourse context: The two lines are also paralleled and balanced, each with four Chinese characters. $>>$ Readers are supposed to appreciate the aesthetic value in such desire and the humor in the personification.

From the above analysis we can see that generally speaking, the presuppositions as aroused by generic and situational contexts in the original are correspondent with those in target texts. However, differences in terms of discourse contextual presuppositions or the different characteristics of target language determine what surface structures are used in translated texts. Of course there may be cases where situational contextual presuppositions also differ between the Chinese and English cultures. Still, under general circumstances, generic contextual presuppositions are most likely to be similar between the two cultures, situational contextual presuppositions the second likely, and discourse contextual presuppositions the least likely to be equivalent. Since in many cases humor is realized via surface linguistic structures, some humorous expressions in the original text can not be reproduced in the target language, and flexible or creative strategies in accordance with target linguistic characteristics may be made use of in translation. Different from humor which to a great degree depends on language forms as just noted, personification is more about the deeper meaning instead of surface forms. We may infer that as personification is more concerned with generic and situational contextual presuppositions, the translation of personification may be quite faithful or straightforward. In the following section we will present the detailed analysis of translating personification. 


\section{Analysis of Personification}

We will analyze the presuppositions in examples (4), (5) and (6) according to the framework as presented in section 3.2.

Example (4):

ST:

(a) Generic context: Good quality or durability is important for tyres. $>>$ People expect good quality or durability of tyres.

(b) Situational context: Personifying the product as a person, who is promised to be able to run the longest. >> Audience may have come across advertisements which praise the good quality of tyres, namely "he who laughs best". Here audience is reminded that durability is more important for tyres.

(c) Discourse context: The idiom "He laughs best who laughs last" is adapted here according to the subject that is being talked about, namely tyres, running, and durability. $>$ Readers are supposed to appreciate the creativity and the humor in the personification.

TT:

(d) Generic context: Good quality or durability is important for tyres. >> People expect good quality or durability of tyres.

(e) Situational context: Personifying the product as a person, who is promised to be able to run the longest. >> Audience may have come across advertisements which praise the good quality of tyres, namely “笑得最好(xiao de zui hao; he who laughs best)". Here audience is reminded that durability is most important for tyres' good quality. 
(f) Discourse context: The idiom "He laughs best who laughs last" is adapted in ST according to the subject that is being talked about, namely tyres, running, and durability, and it is translated literally into Chinese. $>>$ For those who have knowledge about English, they may understand the creativity in the wording; for those who do not, they can still get the humor in the personification.

Example (5):

ST:

(a) Generic context: Watches are expected to be able to run accurately non-stop. $>>$ Consumers appreciate reliability of watches.

(b) Situational context: People all need rest. Rolex watch is personified as a person who does not need any rest. $>>$ Audience needs a reliable watch as companion.

(c) Discourse context: The words are simple, but the personification leaves people a warm and loving impression. >> Readers are supposed to appreciate the implications in the personification.

TT:

(d) Generic context: Watches are expected to be able to run accurately non-stop. $>>$ Consumers appreciate reliability of watches.

(e) Situational context: People all need rest. Rolex watch is personified as a person who does not need any rest. >> Audience needs a reliable watch as companion.

(f) Discourse context: The personification leaves people a warm and loving impression. >> Readers are supposed to appreciate the implications in the personification. 
64 Differences in the Transference of Humor and Personification

Example (6):

ST:

(a) Generic context: It is a romantic thing to visit every corner of the world with one's loving friend. $>>$ Consumers may appreciate such romantic atmosphere.

(b) Situational context: Personifying the Rolex watch as a dependable friend, who can accompany the wearer to visit every corner of the world. $>>$ Audience may have the wish to have such a romantic and loving friend.

(c) Discourse context: The lines are paralleled and balanced. >> Readers are supposed to appreciate the aesthetic value in the wording and the implications of the personification.

TT:

(d) Generic context: It is a romantic thing to visit every corner of the world with one's loving friend. >> Consumers may appreciate such romantic atmosphere.

(e) Situational context: Personifying the Rolex watch as a dependable friend, who can accompany the wearer to visit every corner of the world. $>>$ Audience may have the wish to have such a romantic and loving friend.

(f) Discourse context: The lines are paralleled and balanced, and three of them are composed of six Chinese characters. $>>$ Readers are supposed to appreciate the aesthetic value in the wording and the implications of the personification.

From analysis of examples (4), (5) and (6), we can see that the contextual presuppositions are very much similar between source text and target text in terms of personification. In comparison with the analysis about examples (1), (2) and (3) in terms of humor, the translation of personification is much more direct and faithful, while 
the strategies for translating humor are quite flexible and creative. Faithfulness and flexibility must have their own profound reasons. In the following section, we will explore such reasons with the help of presuppositions we have figured out in each text.

\section{Further Elaboration}

As we have mentioned at the beginning, humor and personification sometimes overlap, especially when humor is realized via personification; however, we are not focusing on their overlap and will explore them separately. For examples (1), (2) and (3), which are to illustrate humor, it is most likely that discourse contextual presuppositions differ (see examples (1), (2)), less likely for the situational presuppositions(see examples (1)), and least likely for generic presuppositions. Within the three examples, only the third one, where the humour lies with the personification, has correspondent presuppositions between original and target texts. Actually, the humorous effect in example (3), similar to examples (4), (5) and (6) about personification, all three categories of presuppositions are equivalent between source and target texts. Here we are not suggesting that every translation of advertisements with humorous or personified expressions follows this pattern; however, this is a common tendency, at least within our corpus. There are three possibilities behind this tendency.

Firstly, as in both cases of translating humor and personification, generic contextual presuppositions are paralleled between Chinese and English texts; it is possible that the relevant world knowledge as involved is universal to both contexts. We dare to claim this, because translators, especially those translating advertising texts, will always consider the target audience, such as their likes, dislikes and needs. This specific goal determines that translators have to respect and attract the target readers in order for the translations to 
66 Differences in the Transference of Humor and Personification

work effectively. Therefore, the high degree of correspondence between generic presuppositions of the Chinese and English texts suggests that the world knowledge of the two cultures is similar. Otherwise, translators would not make the decision to keep the general cultural or moral direction of source texts in translations. Since the two cultures are similar in terms of universal world knowledge, personification in one language can be translated into the other in a direct way, while humor in a more indirect way, which we will illustrate later on. The point is that even when humor is transferred in different ways, the generic contextual presuppositions we have identified are correspondent between source and target texts, as illustrated by analysis in the above section.

Secondly, situational presuppositions are mainly about consumer needs, and translators are quite flexible in dealing with such needs especially when translating humor. From our analysis, in some cases translators hold different situational presuppositions about target audience. This category of presuppositions is not absolutely distinct from generic contextual presuppositions; instead, they are more closely related to the immediate communicative situation than generic ones. Consumer needs are complicated and of many kinds. For illustration, according to Maslow's theory, there are generally three categories of human needs, namely conative, cognitive, and aesthetic needs. Conative needs cover such categories as physiological needs, safety needs, social / love needs, esteem needs, and self-actualization (Muchinsky 2003: 376). The advertising texts are composed in consideration of possible needs of the target audience. However, since sometimes translators may hold different situational presuppositions about the target audience, they will make adjustment accordingly. As generic and situational contexts are common to a degree, although there may be nuanced variation across different cultures. We infer that translators, who are familiar with both cultures, have similar presuppositions about original and target readers when it comes to universal world knowledge and 
communicative situation. Therefore, the translated texts are equivalent to the original texts in terms of personification.

Thirdly, discourse contextual presuppositions are quite different between the Chinese and English languages. We can see that translations of humorous advertisements are usually re-written from the source texts. As mentioned in this paper, personification is more dependent on deeper meaning, while humor is very often realized via language forms including different rhetorical devices such as puns. Linguistic devices rely on language forms to a great degree, so different languages may have different ways to realize the same humorous effects. We hereby infer that translators, who know both languages well, have different presuppositions as to what may be humorous to original and target readers. Since advertisements are intended to be memorable and attractive to readers, faithfulness in terms of textual forms is not that important, and to realize the expected effects is the predominant goal. That is why we have found so many humorous advertisements translated in flexible and creative ways. Different from humor, personification depends more on textual meaning than textual forms. In other words, personification is more about contextual implications which are usually beyond language forms.

\section{Conclusion}

This research, done on the basis of a prior study on humor in translation of advertising texts, proves flexibility in the translation of humor within our corpus. However, when we observe the translation of personification, there is a different tendency because personification is most frequently translated in straightforward ways. Therefore, we start to explore such difference between dealing with humor and personification in translation. Some specific examples are analyzed, and further issues that need addressing in the future study include 
68 Differences in the Transference of Humor and Personification

the following. First, we have come across some difficulty in collecting data and the corpus we have built up is composed of only 98 examples, which is far from enough to represent the most general features of advertisement translation. It is quite easy to find advertisements in English or Chinese, but not that easy to find bilingual advertisements. This is perhaps because there are not so many products or services that are publicized with the same advertisement in both the Chinese and English communities. Second, as to the methodology in this study, we have adopted the concept of presupposition from linguistics, yet use it in a contextual sense. Our argument concerning this point is that, translation, involving at least two languages and cultures, can not be covered by linguistics alone, which usually explores language separately, independent of cultural or contextual influence. We have introduced the notion of presupposition based on the fact that every translator works with his/her presuppositions. In order to make advertisement translations successful, translators have their presuppositions as to the way to arouse the target audience's interest and desire. Third, the shortcomings as just mentioned may lead to criticism concerning what we have summarized about translation of advertisements. We admit that there may be other contrary examples; however, within the corpus we have observed, we find those general rules in translating humor and personification. If possible, we will try to build a larger corpus and test the rules we summarized since we. We believe that the rules are representative to a certain extat.

\section{References}

Cui, G. 1993. Advertising English: 3000 Examples. Beijing: Beijing Institute of Technology Press.

Garner, R. 1983. "Presupposition" in Philosophy and Linguistics. Fillmore, J. \& Langendoen, D Terence. (eds). Studies in 
Linguistics Semantics. New York: Irvington Publishers. Inc.

Givón, T. 1989. Mind, Code, and Context: Essays in Pragmatics.

Hillsdale, New Jersey: Lawrence Erlbaum Associates.

Green, M. 1989. Pragmatics and Natural Language Understanding.

Hillsdale, New Jersey: Lawrence Erlbaum Associates, Publishers, 71-82, 112-114.

Keenan, L. 1998. Two Kinds of Presupposition in Natural Language.

Kasher, A.(ed). Pragmatics: Critical Concepts. London \& New

York: Routeledge, 4, 8 - 15.

Marmaridou, A. 2000. Pragmatic Meaning and Cognition.

Amsterdam \& Philadelphia: John Benjamins Publishing Company.

Mey, J. 2001. Pragmatics: An Introduction. Beijing: Foreign

Language Teaching and Research Press.

Muchinsky, M. 2003. Psychology Applied to Work: An Introduction

to Industrial and Organizational Psychology. Australia; Belmont, CA: Wadsworth Pub..

Sandt, A. van der. 1988. Context and Presupposition. London: Croom Helm.

Segerdahl, P. 1996. Language Use: A Philosophical Investigation into the Basic Notions of Pragmatics. London: Macmillan Press Ltd.

Wu, K. \& H. Zhiwei.(eds.) 1999. An Anthology of Selected English Advertisements. Beijing: Beijing University Press.

Ying Cui

Department of Chinese,

Translation \& Linguistics,

City University of Hong Kong,

Kowloon, Hong Kong

Phone number: 00852-3442-3764; Email: cuiyingcui@163.com

Received Jul. 2010; Reviewed Aug. 2010; Revised version received Aug. 2010. 